\title{
HABILIDADES PARA EL CUIDADO HUMANIZADO DE ESTUDIANTES DE ENFERMERÍA
}

\author{
SKILLS REQUIRED BY NURSING STUDENTS TO PROVIDE HUMANIZED CARE \\ HABILIDADES DE ATENDIMENTO HUMANIZADO DOS ESTUDANTES DE ENFERMAGEM
}

\author{
Azucena Landman Navarro \\ cecilia.landman@uv.cl \\ Rose Marie del Alcazar Pabst \\ Yesenia Madrid Zamorano \\ Leticia Pais Veliz \\ Eitel Rosenkranz Moreno \\ Ivonne Vivanco Lobato
}

Universidad de Valparaíso, Valparaíso, Chile.

\section{RESUMEN}

La formación de estudiantes de enfermería debe potenciar sus habilidades para brindar cuidados adecuados, vinculando experiencias propias, conocimientos científicos, habilidades técnicas, valores y motivación para trabajar por y para otros. Existe riesgo de deshumanización debido a los modelos institucionales administrativos y técnicos de los centros de salud. El cuidado humanizado propende al desarrollo de una relación transpersonal, considera valores y necesidades superiores de personas que vivencian el proceso de salud, enfermedad y el bien morir. El objetivo de este trabajo fue identificar habilidades para brindar cuidado humanizado desde la perspectiva de estudiantes. Se trata de un estudio descriptivo y transversal con una población de 98 estudiantes de enfermería de $3^{\circ}$ y $4^{\circ}$ año $\left(n=57 ; 3^{\circ}=20 ; 4^{\circ}=37\right)$ a los que se les solicitó consentimiento informado así como también autorización a la coordinación de la carrera. Se utilizó como instrumento el Inventario de habilidades del cuidado, el cual mide las áreas de Conocimiento, Valor o Coraje y Paciencia correspondientes a un valor $\alpha$ de Cronbach de 0.74 . Se utilizaron estadígrafos de distribución de frecuencias, media de datos agrupados, promedios y porcentajes (Software SPSS-15). Los resultados muestran que se obtuvo un resultado de $63 \%$ en Paciencia, un $35 \%$ en Conocimiento y sólo un $2 \%$ en Valor-Coraje. La Paciencia requiere del ejercicio de tolerancia, respeto y adaptación frente a la incertidumbre situacional, generándose un vínculo estrecho entre la persona y el estudiante; fue la habilidad mejor evaluada. El Conocimiento incluye autoconocimiento y conocimiento del otro; está menos desarrollado, provocando una brecha en el acto de cuidar. El bajo resultado en Valor-Coraje señala la dificultad para enfrentarse a lo desconocido y en la propia capacidad de cuidar, influyendo negativamente en el desarrollo de la fuerza necesaria para afrontar el cuidado adecuado. Estos resultados muestran la necesidad de fortalecer en estudiantes de enfermería habilidades de afrontamiento ante situaciones cotidianas emergentes, especialmente las dimensiones de Conocimiento y de Valor.

Palabras Clave: Educación, Empatía, Enfermería, Aptitud, Competencia Clínica.
Recibido: 15/01/2016

Aceptado: 05/04/2016

\begin{abstract}
Training of nursing students should enhance their skills to provide proper care, linking experience, scientific knowledge, technical skills, values and motivation to work with and for others. There is a risk of dehumanization due to administrative and technical health centers institutional models. Humanized care tends to develop a transpersonal relationship, considers values and higher needs of people who go through the process of health, disease and dignified death. The aim of this study was to identify skills to provide humanized care from the perspective of students. It is a descriptive cross-sectional study with a population of 98 nursing students of $3 \mathrm{rd}$ and 4 th year $(n=57$; $3 \mathrm{rd}=20 ; 4$ th $=37$ ) whose consent was requested, and also the authorization of the school authorities. The instrument used was the Care Ability Inventory (CAI), which measures the areas of Knowledge, Courage and Patience corresponding to a value of Cronbach $\alpha$ of 0.74 . Statistics like frequency distribution, mean, average and percentages were used (Software SPSS-15). The results show a score of $63 \%$ in Patience, 35\% Knowledge and only $2 \%$ in Courage. Patience requires the exercise of tolerance, respect and adaptation to situational uncertainty, generating a close link between the person and the student; this ability scored the highest. Knowledge includes self-knowledge and knowledge of others; it is less developed, causing a breach in the act of caring. The low value in Courage shows the difficulty in facing the unknown and in one's ability to care, negatively influencing the development of the necessary strength to face proper care. These results show the need to strengthen nursing students coping skills to everyday situations, especially the dimensions of Knowledge and Courage.
\end{abstract}

Keywords: Education, Empathy, Nursing, Clinical Competence.

\section{RESUMO}

A formação acadêmica deve potencializar habilidades para oferecer cuidados dos estudantes de enfermagem, vinculando experiências próprias, conhecimentos científicos, habilidades técnicas, valores, motivação para trabalhar por e para outros. 
Existe risco de desumanização, devido aos modelos institucionais administrativos e técnicos dos centros de saúde. O cuidado humanizado busca o desenvolvimento de uma relação transpessoal, e considera os valores e necessidades superiores das pessoas que vivenciam o processo de saúde, doença e morrer. $\mathrm{O}$ objetivo foi identificar habilidades para oferecer cuidado humanizado, da perspectiva dos estudantes. Trata-se de um estudo descritivo e transversal com uma população de 98 estudantes de Enfermagem do 3으 e 4ㅇ ano ( $n=57 ; 3$ 으 = $20 ; 40=37$ ), aos quais foi solicitado consentimento informado; também foi pedida autorização à Coordenação do curso. Utilizou-se o "instrumento inventário habilidades de cuidado", que mede Conhecimento, Valor ou Coragem e Paciência correspondente a $\alpha$ de Cronbach 0,74 . Foram utilizados estratigrafias de distribuição de frequências, média dos dados agrupados, médias, porcentagens (software: SPSS-15). Os resultados obtiveram Paciência 63\%, e Conhecimento $35 \%$; Valor só obteve $2 \%$. Concluiu-se que Paciência requer o exercício da tolerância, respeito e adaptação diante da incerteza situacional, habilidade melhor avaliada, gerando um vínculo estreito entre a pessoa e o estudante. $\mathrm{O}$ conhecimento inclui autoconhecimento e conhecimento do outro; desenvolvida em menor escala, provocando uma lacuna no ato de cuidar. No desenvolvimento do valor ou coragem, indica dificuldade para enfrentar o desconhecido e à própria capacidade de cuidar, influindo de forma negativa no desenvolvimento da força necessária face ao cuidado. Esses resultados mostram a necessidade de fortalecer nos estudantes de Enfermagem suas habilidades para enfrentar situações cotidianas emergentes, especialmente as dimensões de Conhecimento e Valor.

Palavras-chave: Educação, Empatia, Enfermagem, Aptidão, Competência Clínica.

\section{INTRODUCCIÓN}

En Chile las instituciones formadoras explicitan perfiles de egreso orientados a la consecución de un profesional generalista, crítico, reflexivo y humanista; diseñan planes de estudios y evaluaciones en distintas actividades curriculares. Sin embargo, se desconoce la perspectiva de los estudiantes de enfermería en cuanto al desarrollo de habilidades psicosociales, actitudinales y relacionales, necesarias para brindar un cuidado humanizado $(1,2)$. Por ello, este estudio tuvo como objetivo medir las propias habilidades de cuidado de estudiantes de tercer y cuarto nivel de enfermería, pertenecientes a una institución de educación superior, quienes han realizado diversas prácticas de cuidado durante el año 2014. El sentido de este estudio ha sido disponer de un diagnóstico de la formación de pregrado en Enfermería, haciendo más palpable la integración de las prácticas de cuidado y de la gestión con las bases teóricas del ser humano y el cuidado humanizado, a través de la relación transpersonal estudiante-paciente, según la teoría de Jean Watson $(3,4)$.

Para aproximarse al fenómeno de estudio, habilidades para el cuidado, es necesario reconocer primeramente el carácter sub- jetivo del cuidado, que se desarrolla a través de una relación transpersonal, en interacción con un otro importante; sin embargo, en este caso se ha utilizado un instrumento cuantitativo, el Inventario de Habilidades de Cuidado (Care Ability Inventory, CAI) el cual mide algunos elementos teóricos del cuidado humanizado, el conocimiento, el valor o coraje y la paciencia, como una manera de concreción de un concepto abstracto y de disponer de evidencias del nivel de existencia como fenómeno investigativo $(5,6)$. El cuidado ha sido tema central de reflexión para diversas teoristas de enfermería y de la discusión filosófica, $(4,7,8)$. Éstos señalan que el cuidado presupone una interacción entre seres humanos, que implica confianza básica, misericordia y expresión debida para cuidar a personas vulnerables; toma en cuenta sus intereses, abstrayéndose del individualismo y del relativismo ético $(9,10)$.

El cuidado como expresión de una cualidad humana se manifiesta en una disposición moral orientada hacia la ayuda y el compromiso. Es una experiencia que va más allá de atender un conjunto de desequilibrios biológicos de las personas, sino que considera las dimensiones psíquicas, socioculturales y espirituales (11-13). Uno de los aspectos relevantes de la formación es que todo estudiante de enfermería no sólo debiera demostrar conocimientos teóricos, habilidades y destrezas en el desarrollo de las actividades prácticas, sino que también debería brindar un cuidado integral, establecer relaciones de ayuda mutua, ser tolerante, tener sensibilidad, demostrar respeto y compasión, tratar con dignidad, generar confianza y demostrar amor a las personas (14-15).

El fundamento para realizar el estudio ha sido la existencia de elementos contextuales en la atención de salud que ponen en riesgo la humanización del cuidado, es decir, la pérdida de sus atributos y de la dignidad humana al interior de la relación entre el profesional de salud y el paciente (16-18). Estudios revisados muestran que tanto en Chile como en otros países de Latinoamérica el cuidado se desarrolla en un escenario dual entre un modelo biomédico, basado en las enfermedades, y un modelo humanista, cuyo foco está puesto en las respuestas humanas (19-21). Sin desconocer la relevancia del desarrollo científico y tecnológico, que beneficia la atención clínica de las personas, existe el riesgo de una mecanización involuntaria por parte del equipo de salud, descuidando el vínculo necesario entre la atención técnica y un cuidado humano holístico (2224). Las personas que acuden a los centros de salud valoran más la calidez de los cuidados y la comunicación que la tecnología aplicada a éstos $(25,26)$.

Existen otros factores que ponen en riesgo la humanización del cuidado, tales como las condiciones laborales, la fragmentación de los procesos clinicos y de gestión, las remuneraciones, la sobrecarga de trabajo, las escasas instancias de discusión y de reflexión entre profesionales y las limitadas competencias del profesional para asumir una atencion humanizada (27). Estudios realizados en Chile y en el extranjero abren la posibilidad de identificar las habilidades que los estudiantes señalan como necesarias para brindar cuidado, sin reducirlas sólo al área instrumental; deben contar con habilidades que forman 
parte de su estructura psicosocial, que se reflejará de manera negativa o positiva en un actuar frente al paciente y familias $(12,14,16)$. Tradicionalmente los programas de estudio no se organizan de manera integrada entre las ciencias humanas y las derivadas de las ciencias básicas, lo cual dificulta una comprensión de los procesos involucrados en la relación humana; junto con ello existe una mayor distribución de carga horaria hacia el logro de habilidades técnicas y una menor disposición para actividades de carácter humanístico y reflexivo $(13,15)$. En el contexto de la docencia en Enfermería, guiada por aspectos ontológicos, valóricos y epistemológicos del ser humano en interacción con otros, los estudiantes intentan construir la forma de brindar cuidados a través de sus experiencias formativas; es por ello que este estudio se ha planteado como objetivo identificar las habilidades para brindar cuidado humanizado desde la perspectiva de los estudiantes.

\section{METODOLOGÍA}

Se realizó un estudio descriptivo, de corte transversal, en una institución privada de educación superior de la ciudad de Valparaíso, Chile, en abril del año 2014. La población corresponde al universo de estudiantes de Enfermería de $3^{\circ}$ y $4^{\circ}$ nivel; $\mathrm{N}=98$. Se selecciónó una muestra condicionada a aceptación para participar y al cumplimiento de criterios de inclusión: ser estudiantes de $3^{\circ}$ y $4^{\circ}$ nivel y haber realizado prácticas de cuidado, correspondiendo al $58 \%$ de la población, $n=57\left(3^{\circ}=\right.$ $20 ; 4^{\circ}=37$ ). Se obtuvo la autorización de la coordinación de carrera de Enfermería; firma, consentimiento informado, respetando compromiso, anonimato y confidencialidad de datos.

Se utilizó el Instrumento "Inventario de habilidades de cuidado", desarrollado por Ngozi Nkongho (Caring Ability Inventory, CAI) y la autorización de la Dra. Jean Watson para su utilización, traducción y modificación según el contexto cultural. El mismo consta de 37 preguntas, con respuestas medidas con Escala Likert 1-7; las puntuaciones más altas indican un mayor nivel de cuidado si el ítem está redactado de manera positiva; la puntuación se revierte si el ítem está redactado de forma negativa. El CAI permite medir, analizar y comprender las relaciones humanas, identificando las áreas de debilidad y potencialidad en el cuidado de una persona. Las variables son: Conocimiento, Valor o Coraje y Paciencia (Tabla $\mathrm{N}^{\circ} 1$ ). La fase de validación CAI contó con la traducción y retro traducción por tres personas bilingües, con lengua materna español, usada en Chile. La versión resultante fue revisada por un traductor de lengua materna inglesa, quien desconocía la versión original. Esta nueva versión fue evaluada con técnica Delphi por ocho expertos enfermeros, docentes y clínicos. Al instrumento resultante de esta validación, se le hicieron los ajustes pertinentes, evaluando su utilidad, claridad y representación de cada dimensión. La validación facial de contenido y de criterio fue realizada por la autora del instrumento (5).

Se realzó una prueba piloto de comprensión aplicándose a una muestra de juicio a ocho estudiantes que no se consideraron en el estudio final (equivalente a $9 \%$ de la muestra) con evalua- ción dirigida a la comprensión de cada ítem y la ambigüedad de los términos usados.

La consistencia interna del instrumento obtuvo una confiabilidad global, $\alpha$ Cronbach 0.74 . Nunnally J. y Bernstein establecen que para estudios exploratorios dicho valor se considera de una fiabilidad aceptable, muy similar a la obtenida por Nkongho $(5,28)$.

Tabla № 1: Variables medidas del instrumento (5)

\begin{tabular}{|l|l|}
\hline Variables & Definición conceptual \\
\hline Valor & $\begin{array}{l}\text { Habilidad para enfrentarse a lo desconocido. } \\
\text { El valor para cuidar se obtiene de experiencias } \\
\text { del pasado, siendo sensible y abriéndose a las } \\
\text { necesidades del presente. Se debe confiar en el } \\
\text { crecimiento del otro y en la propia capacidad de } \\
\text { cuidar. }\end{array}$ \\
\hline Conocimiento & $\begin{array}{l}\text { Condición primordial para cuidar, incluye el } \\
\text { autoconocimiento, el conocimiento del otro, } \\
\text { sus fortalezas y limitaciones al igual que sus } \\
\text { necesidades particulares; y el ritmo alternativo } \\
\text { está relacionado con las fluctuaciones o cambios } \\
\text { que se dan durante la experiencia del cuidado. }\end{array}$ \\
\hline Paciencia & $\begin{array}{l}\text { Tolerante en situaciones donde se representa } \\
\text { cierto grado de confusión y de desorganización. } \\
\text { Es la actitud que le permite a la persona poder } \\
\text { soportar cualquier contratiempo y dificultad. }\end{array}$ \\
\hline
\end{tabular}

Para la determinación de los niveles de habilidad de cuidado se asignó valores de bajo, medio y alto, basada en el estudio Nkongho, ajustada por investigadores, como se muestra en la Tabla № 2.

Tabla № 2: Caring Ability Inventory. (5)

(Adaptación por investigadores del estudio, promediando valores, sin diferenciación de sexo)

\begin{tabular}{|c|c|c|c|}
\hline Eariables & Bajo & Medio & Alto \\
\hline Paciencia & $<55.7$ & $55.7-63.5$ & $>63.5$ \\
\hline Conocimiento & $<66.7$ & $66.7-77.3$ & $>77.3$ \\
\hline Valor o coraje & $<58.2$ & $58.2-69.8$ & $>69.8$ \\
\hline
\end{tabular}

Junto con el CAI se aplicó encuesta de caracterización de la muestra, con las variables de edad, sexo, nivel, jornada y año de ingreso a la carrera. El procesamiento de datos se realizó con el programa SPSS versión 15 y los estadígrafos utilizados fueron distribución de frecuencias, media de datos agrupados, promedios, porcentajes.

\section{RESULTADOS}

Caracterización de los estudiantes: $86 \%$ mujeres; 65\%, mayores de 25 años; $65 \%$, pertenecen al $4^{\circ}$ nivel de la carrera; 
$51 \%$ ingresó a la carrera entre los años 2011 y 2012; $40 \%$ entre 2009-2010; 60\% corresponden a jornada diurna y $40 \%$ a jornada vespertina.

El resultado global de las habilidades de cuidado reporta un predominio de la variable Paciencia, siendo la segunda habilidad el Conocimiento; muy por debajo se ubica el Valor o Coraje (Gráfico $N^{\circ} 1$ ).

\section{Gráfico $N^{\circ} 1$ Distribución habilidades globales.}

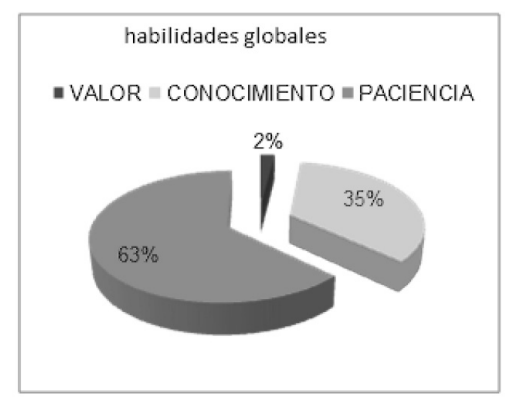

No encontrándose diferencias por edad, sexo o niveles cursados (Gráficos $N^{\circ}$ 2, 3 y 4).

Gráfico $\mathrm{N}^{\circ}$ 2: Distribución de habilidades de cuidado según edad.

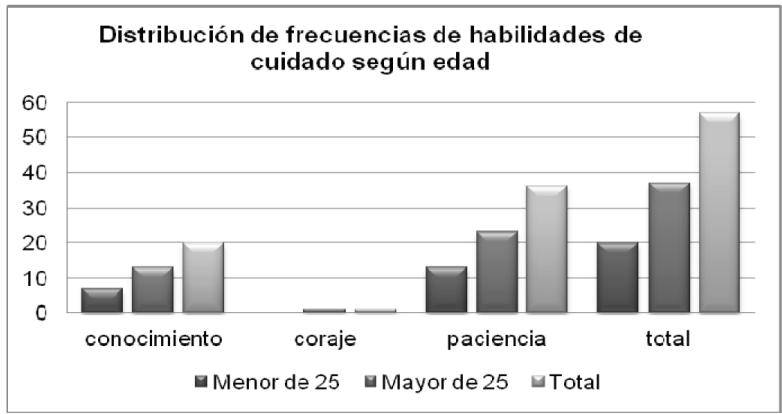

Gráfico N³: Distribución de habilidades de cuidado según nivel que cursan.

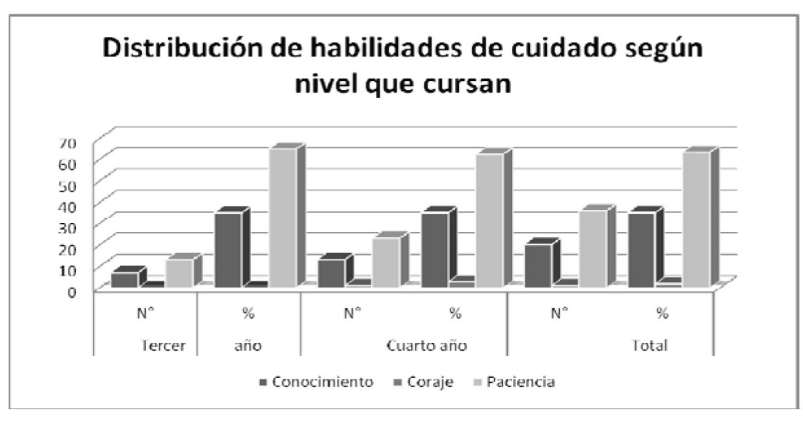

Se observó un incremento progresivo de habilidades de cuidado en las nuevas cohortes; los estudiantes de la jornada vespertina presentan valores más altos en cuanto a la variable Paciencia, no así en Conocimiento, que es mayor en la jornada diurna; sin embargo, ambos grupos coinciden en los bajos valores presentados en la variable Coraje.

\section{DISCUSIÓN Y CONCLUSIONES}

Los estudiantes que formaron parte de esta investigación son personas únicas, con habilidades y herramientas distintas y variadas, pero con un objetivo en común: entregar un cuidado de calidad y humanizado. Desde el origen de la humanidad, las prácticas humanas relacionadas con la salud y el cuidado de las personas se han considerado una actividad femenina; actualmente existen enfoques de género que critican esta posición y el rol social de la mujer, teniendo en cuenta que cada vez más se incrementa el ingreso de hombres a estudiar la carrera (29). La edad de los estudiantes en promedio es de 25 años, en una etapa de productividad y de reflexión propia de la adultez temprana, que tienen decidido su plan vital y profesional, con una madurez psicológica para el autoconocimiento de propias debilidades y potencialidades. Por estar en una etapa vital han desarrollado un sistema de valores, sentido de la autonomía, autocontrol y responsabilidad que los lo habilita para brindar cuidados de manera integral (30).

De manera global, las habilidades para el cuidado humanizado presentaron un predominio de la habilidad Paciencia, seguido del Conocimiento, siendo el Valor o Coraje una habilidad menos lograda. Al comparar los resultados de este estudio con los obtenidos por la autora del instrumento, los participantes estudiados presentarían un nivel medio de Paciencia, casi en el límite del nivel alto (5). En cuanto al Conocimiento se encontraría en un nivel bajo, siendo éste muy menor al estudio antes mencionado con valores < 66.7. Más notoria aún es la diferencia respecto al Valor, que se encuentra en niveles muy bajos, < 58.2. La Paciencia reportó un nivel medio, lo que significa buen nivel de tolerancia y comprensión de quien se cuida, aspectos de gran importancia para los estudiantes de enfermería, quienes poseen atributos para asumir una actitud que facilite una comunicación flexible, cálida, expectante, neutral, desprovista de comportamientos autoritarios y centrada en lo que acontece al otro, favoreciendo la cooperación del paciente y facilitando la relación intersubjetiva por parte de éste $(4,12,18)$. Al comparar con otro estudio realizado a enfermeras/os colombianas/os, los estudiantes encuestados mostraron mejor nivel, pudiéndose explicar que en su calidad de aprendices disponen de mayor tiempo para la comunicación, asumiendo un rol menos autoritario, en una relación más horizontal $(18,31)$. Esto implicaría un mayor ejercicio de la tolerancia y ajustes frente a la incertidumbre situacional, generándose un vínculo estrecho y gratificante entre la persona y el estudiante $(3,4,19)$.

El Conocimiento propio y del otro se encuentra en un nivel medio bajo, lo cual podría limitar la relación interpersonal, ya que ésta se da en una relación dialógica entre el futuro profesional y el paciente $(8,18,27)$. La formación, como un sistema de valores humanistas y altruistas que se aprenden precozmente, debe ser potenciada a través de modelos aprehendidos en la relación transpersonal con enfermeras/os clínicas y docentes, quienes por su experiencia han logrado mejores niveles en esta dimensión $(18,32)$. El aprendizaje transpersonal es una herramienta de significado para relacionarse de manera genuina con 
las personas que requieren de información y conocimientos respecto a su estado de salud y cuidados necesarios $(3,18,20)$.

En cuanto al Valor o Coraje, definido como una habilidad para enfrentarse a lo desconocido, confiando en el crecimiento del otro y en la propia capacidad de cuidar, en este estudio se lo observa como una habilidad ubicada mayoritariamente en los más bajos niveles, coincidiendo con estudios realizados a profesionales de enfermería y a cuidadores familiares, lo cual podría reflejar una actitud más bien pasiva, poco optimismo, temerosos y con algunas carencias para comprender el dolor ajeno $(13,33)$. El ejercicio del valor a través de la formación abre un campo al cual hay que poner atención, toda vez que este es fundamental para establecer una relación de ayuda (10, $18,23)$.

Es necesario potenciar a través de la formación las habilidades de cuidado, preparando a los estudiantes para que desarrollen un genuino interés por los otros; que tengan el conocimiento necesario para que sus acciones sean eficaces y oportunas; que reconozcan sus propias limitaciones y puedan encontrar las herramientas psicoemocionales para la superación personal.

\section{CONSIDERACIONES FINALES}

El estudio permitió tener una visión particular del cuidado humanizado de acuerdo a la teoría de Jean Watson, reflexionar acerca de nuestras propias competencias docentes para enseñar el cuidado, focalizándose hacia el desarrollo personal y el autoconocimiento por parte de los de los estudiantes, que ejercite las cualidades del valor y coraje como preparación para un futuro desempeño como profesionales y que puedan asumir el cuidado en todas sus dimensiones.

Este estudio podría motivar a otros investigadores a realizar investigaciones con enfoque fenomenológico, identificar en profundidad el sentido y significado del cuidado, además de disponer de otras perspectivas del mismo, con la mirada del profesional que gestiona los cuidados, como "ser cuidador" y del paciente como "ser de cuidados".

\section{REFERENCIAS BIBLIOGRAFICAS}

1. Casate J, Corrêa K. A humanização do cuidado na formação dos profissionais de saúde nos cursos de graduação. Rev. Esc. Enferm. USP [Internet]. Feb 2012 [citado 30 Abr 2016]; 46 (1): 219-226. Disponible en: http:// www.scielo.br/scielo.php?script=sci_arttext\&pid=\$0080 62342012000100029\&Ing

2. Garavito A. La formación integral de estudiantes de enfermería. Investig. Enferm. Imagen Desarr. 2013;15(2):153172.

3. Watson J. Human caring science: a theory of nursing. 2 da ed. Sudbury: Jones \& Bartlett Learning; 2012.

4. Watson J. Nursing: the philosophy and science of caring. 2da ed. Boston: University Press of Colorado; 1985.
5. Watson J. Assessing and measuring caring in nursing and health science. 2da ed. New York: Springer Publishing; 2002.

6. Vélez C, Huber J. Epistemología del cuidado en enfermería: a la luz de la fenomenología. Cultura del cuidado Enfermería. 2011; (1): 52.

7. Levinas E. Humanismo del otro hombre. 6a ed. Madrid: Siglo XXI; 2006.

8. Leininger M. Caring: an essential human need. En: Proceedings of the Three National Caring Conference. Detroit: Michigan: Waine State University;1988.

9. Waldow V. Cuidado humano: La vulnerabilidad del ser enfermo y su dimensión de trascendencia. Index de enfermería/cuarto trimestre 2014, vol. 23, N. 4

10. Rivera L, Triana Á. Cuidado humanizado de enfermería: visibilizando la teoría y la investigación en la práctica, en la clínica del Country. Actual Enferm. 2007;10(4):15-21.

11. García L. La ética del cuidado y su aplicación en la profesión enfermera. Acta Bioethica 2015; 21 (2): 311-317

12. Beltrán O. Atención al detalle, un requisito para el cuidado humanizado. Index Enferm [Internet]. 2015 [citado 08 May 2016]; 24 (1-2): 49-53. Disponible en: http:// dx.doi.org/10.4321/S1132-12962015000100011.

13. Duarte M., Noro A. Humanização: uma leitura a partir da compreensão dos profissionais da enfermagem. Rev. Gaúcha Enferm. [Internet]. Dic 2010 [citado 30 Abr 2016]; 31(4): 685-692. Disponible en: http://dx.doi. org/10.1590/S1983-14472010000400011.

14. Rivas E, Rivas A, Bustos L. Valores expresados en estudiantes de enfermería. Cienc Enferm. 2011; XVII (2): 65-75.

15. de Souza J. Lima R., Munari, D. Esperidião E. Ensino do cuidado humanizado: evolução e tendências da produção científica. Rev. Bras. Enferm. [Internet]. 2008 [citada 30 Abr 2016]; 61(6): 878-882. Disponible en: http://www. scielo.br/scielo.php?script=sci_arttext $\&$ pid=S003471672008000600014\&Ing

16. Rigon N., Junges J. Politica de humanizacao na atencao basica: revisao sistematica. Rev. Saúde Pública. [Internet]. Dec 2013 [citada 30 Abr 2016]; 47(6): 1186-1200. Disponible en: http://www.scielosp.org/scielo.php?script=sci_ arttext\&pid=S0034-89102013000901186\&lng=en .

17. Salgado J, Valenzuela S, Sanhueza O. Comportamientos del cuidado percibidos por estudiantes de enfermería y receptores del cuidado. Cienc Enferm. 2015;1(XXI): 69-79.

18. Contreras A, et al. Habilidad de cuidado de los profesionales de enfermería de una institución prestadora de servicios de salud. Cultura del Cuidado Enfermería. 2011; 8(1): 17-23.

19. Arredondo C, Siles J. tecnología y humanización de los cuidados: una mirada desde la teoría de las relaciones interpersonales. Index Enferm. 2009 ;18(1):32-36.

20. Ceballos A. Desde los ámbitos de la enfermería analizando el cuidado humanizado. Cienc enferm. 2010;16(1): 31-35.

21. Poblete $\mathrm{M}$, Valenzuela S. Cuidado humanizado: un desafio para las enfermeras en los servicios hospitalarios. Acta Paul Enferm. 2007;20(4);4 99-503. 
22. Hackspiel M. El cuidado de una vida humana. Cuadernos de Bioética. 1999; (37):160-167.

23. Neusa, C., Célia R. Humanização e trabalho na enfermagem. Rev. bras. enferm. [Internet]. Abr 2003 [citado 30 Abr 2016]; 56 (2): 189-192. Disponible en: http://www. scielo.br/scielo.php?script=sci_arttext\&pid=S003471672003000200016\&lng=pt.

24. Espinosa Á, Enríquez C, Leiva F, López M, Castañeda L. Construcción colectiva de un concepto de cuidado humanizado en enfermería. Cienc. enferm. [Internet]. Ago 2015 [citado 08 May 2016]; 21 (2): 39-49. Disponible en: http://www.scielo.cl/scielo.php?script=sci_ arttext\&pid=S0717-95532015000200005\&lng=es. http://dx.doi.org/10.4067/S0717-95532015000200005.

25. Bautista R. Percepción de la calidad del cuidado de enfermería en la ESE Francisco de Paula Santander. Aquichán. 2008;8(1):74-84.

26. Landman C. et als. Satisfacción usuaria respecto a competencia de comunicación del profesional de enfermería. Cienc Enferm. 2015; 1(XXI):97-99.

27. Stein D, Santos M, Lorenzini A. Humanizando el cuidado a traves de la valorización del ser humano: resignificación de los valores y principios por los profesionales de salud. Rev. Latino-Am. Enfermagem [Internet]. 2007 [citado 09 Oct 2015]; 15(1): 34-41. Disponible en: http:// www.eerp.usp.br/rlae.
28. Nunnally, J. y Bernstein, Y. Teoría psicométrica. 3a ed. México: McGraw-Hill; 1995.

29. García A. Ma., Sainz A., Botella M. La enfermería vista desde el género. Index Enferm. [Internet]. Nov 2004 [citado 07 May 2016]; 13(46): 45-48. Disponible en: http:// scielo.isciii.es/scielo.php?script=sci_arttext\&pid=S113212962004000200009\&lng=es.

30. Papalia, D.; Wendkos, S. y Duskin, R. Desarrollo humano. Vol. XI ed. México: Mc Graw Hill. 2010. 422-423

31. Ayala R. Biopoder: el poder y la violencia en la formación de profesionales de enfermería. Educ. méd. [Internet]. Sep 2008 [citado 07 May 2016]; 11(3): 125-129. Disponible en: http://scielo.isciii.es/scielo.php?script=sci_ arttext\&pid=S1575-18132008000300004\&Ing=es.

32. González A, de Almeida M. Integralidade da saúde: norteando mudanças na graduação dos novos profissionais. Ciênc. saúde coletiva [Internet]. May 2010 [citado 30 Abr 2016]; 15(3): 757-762. Disponible en: http://www. scielo.br/scielo.php?script=sci_arttext \&pid=S141381232010000300018\&Ing

33. Barrera L, et als. La habilidad de cuidado de los cuidadores familiares de personas con enfermedad crónica. Rev. Inv. \& Educ. en Enf., vol. XXIV, núm. 1, marzo, 2006, pp. 36-46 